\title{
A Research on Structured Analysis of Biomaterial of Dental Implant in Computer Aided Biomechanical Engineering Modelling
}

\author{
Ö. KARAÇALI* \\ Department of Mechanical Engineering, Faculty of Engineering, Istanbul University, Avcılar, Istanbul, 34320 Turkey
}

\begin{abstract}
The research explained in this paper is about the mechanical principle applications on regulated form and function, forces, motion of teeth and dental implant — influencing the implant length and bone quality — in biomechanics engineering modeling in dentistry. The non-linear finite element method was employed as an advanced computer technique of structural stress analysis tool for biomechanics modeling using mechanical, mathematical, and biological definitions and concepts. A finite element model of dental implant with accurate geometry and material properties was developed to make realistic investigations on the implant biomaterial properties and mechanical behavior of new dental implant. The finite element models with non-linear contact elements were used to simulate an interface fixation within the implant system and the sliding function of the non-rigid connector. This research showed that implant design influences force transmission characteristics in peri-implant bone and mechanical signals affect bone tissue differentiation. Hence, it is important to control biomechanical loads on dental implants to maintain osseointegration and to promote early bone-implant interface healing. The results of this analysis are helpful for implant biomaterial selection and design for clinical interest.
\end{abstract}

DOI: $10.12693 /$ APhysPolA.125.186

PACS: 83.50.-v, 87.85.gp, 02.70.Dh

\section{Introduction}

Biomechanics is one of the main factors for achieving long-term success of implant supported prostheses. Dental implants are used to provide mechanical support to a superimposed and crown, for daily chewing loads. Long-term failures mostly depend on biomechanical complications. It is important to distinguish the effects of macro design of the implants [1]. Over the past three decades, significant advances have occurred in the clinical use of oral and maxillofacial implants. Statistics on the use of dental implant reveal that about 300,000 dental implants are placed per year [2]. Dental implants are currently used to replace missing teeth, to rebuild the craniofacial skeleton, to provide anchorage during orthodontic treatments and even to help new bone formation in the process of distraction osteogenesis [3]. Biocompatible titanium and titanium alloys materials are used for dental implants for their excellent corrosion resistance and suitable mechanical properties [4]. Although titanium implants available commercially at present have got many disadvantages such as mismatches between the elastic modulus of the implant and of the bone, different bonding strength between the implant and the bone [5]. A stress shielding or concentration can be easily induced on the interface and results in a potential risk to the long-term stability of the implant [6]. The success or failure of an implant is determined by the manner how the stresses at the bone-implant interface are transferred

*e-mail: ozdogank@istanbul.edu.tr to the surrounding bones. It is important to comprehensively navigate the various factors controlling the success of dental implants [7].

Computational model in this research has been used to better understand many factors affecting the biomechanical behavior. The model proposed in this study and the experiments that were performed highlight the fact that the non-linear behavior of the implant is not only due to its mechanical properties but also due to its geometry. As a consequence, for further investigations, it seems important to take the specific geometry into account. The use of a linear constitutive law can simplify the numerical models, and consequently decrease the computation time.

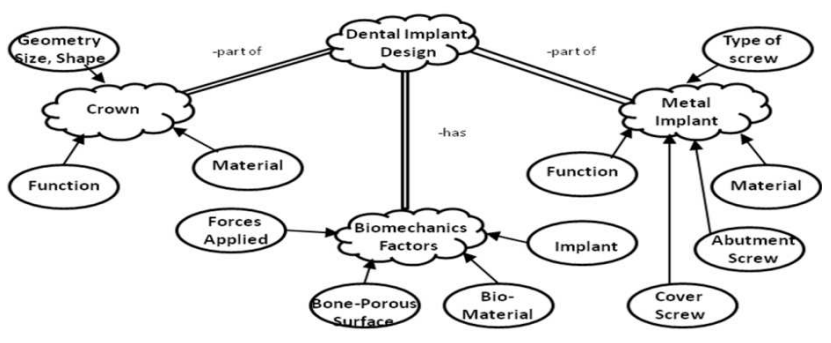

Fig. 1. Research concept area: design factors for dental implant in biomechanical engineering of dentistry.

A dental implant has a body with a press fit diameter to fit into a hole drilled into a patient's jaw bone, and has a helical groove machined into the body diameter to improve the rate of growth of bone tissue therein to securely lock the implant in position [8]. The purpose of this study was to investigate the implant biomechan- 
ics factors: the stress distribution, forces applied, and control of loads on dental implants. Research concept is demonstrated as design factors for dental implant in biomechanical engineering of dentistry in Fig. 1.

\section{Implant design: materials and method}

During the application of the dental implant all processes are followed as shown in Fig. 2. First metal implant is located, then abutment screw is placed. Crown prosthesis is situated on top of the whole implant system. The biomaterial surface interacts with water, ions and numerous biomolecules after implantation. The nature of these interactions, such as hydroxylation of the oxide surface by dissociative adsorption of water, formation of an electrical double layer and protein adsorption and denaturation, determine how cells and tissues respond to the implant [9]. The interactions in implant model aimed to provide a good surface quality and optimum mechanical strength.

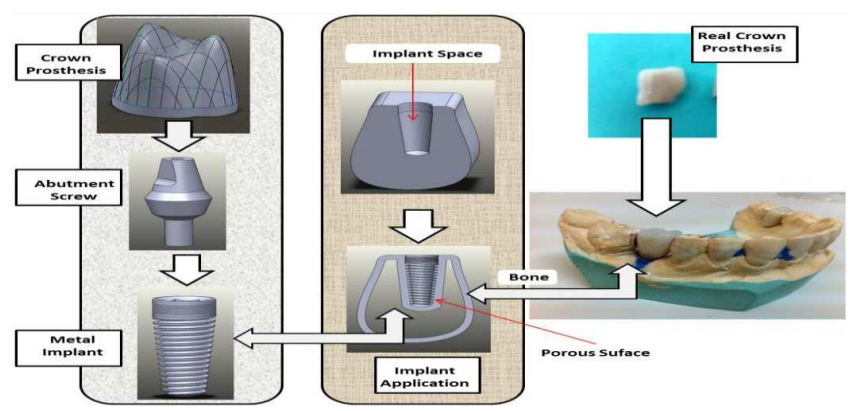

Fig. 2. Application of the dental implant.

An analysis was started from the application of the load on the implant. As a result of an analysis, the distribution of stresses over the contact surfaces of both components of the implant was demonstrated in Fig. 2. It was observed that the stresses developed during both conditions were well within the limits and the implant was reliable for use in human beings. This study has developed a series of predicative finite element analyses [10] of implant design, which incorporate the implant, crown, screw to model the mechanical characteristics of all three layers as shown in Fig. 2.

In Fig. 3, a model of a morse-taper oral implant and a solid abutment was constructed separately. The implantabutment complex was embedded in a $\varnothing 1.4 \mathrm{~cm}$ acrylic resin cylinder. Vertical and oblique forces of $55 \mathrm{~N}$ were applied on the abutment and solved by two different analyses. First, contact analysis was performed in the implant-abutment complex to evaluate implant. Then, the components were bonded with a separation force of $15 \mathrm{~N}$ to analyze an implant.

The applied forces to the implants, developed moments and its directions, and preloaded forces are presented in Fig. 3. An implant in this research is defined as a mechanical system designed to transmit occlusal forces on

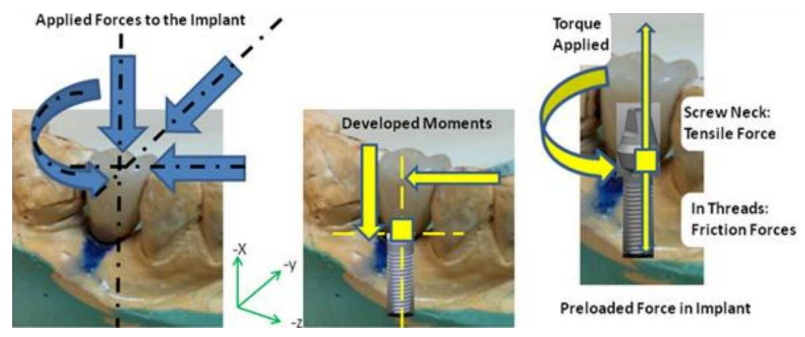

Fig. 3. Applied biomechanical forces research in real dental implant.

a prosthesis through an abutment and implant to the surrounding bone. Titanium materials were assumed to be homogeneous, isotropic and linearly elastic in this research [7]. The bone-implant interfaces were assumed to be completely osseointegrated. The sides and bottom of bones were set to be completely constrained, and the boundary conditions were extended to the corresponding node. Multi-constraining was imposed on implant to limit the freedom of the roots. The forces produce moments, about the screw joint in the screwed abutments. The vertical forces act about the centerline of the implant. Horizontal or lateral forces act about the plane of the abutment and implant interface. Dynamic loading was loaded to evaluate the implant-bone model. The implants were assumed to be under an axial force of 55$200 \mathrm{~N}$ and a lingual force of $30 \mathrm{~N}$ in the angle of approximately $45^{\circ}$ to the occlusal plane. The relationship between applied torque and preload depends on several factors: material properties, particularly stiffness, surface texture, and condition of the mating surfaces, etc.

The stress distribution in the bone zone of the implants is represented in Fig. 4. There are high stress zones in the junction of the porous layer and the dense body. In the bone zone, the interface stress decreases from top to bottom, and increases at the root apex. Figure 5 shows that the maximum stress magnitude to complete the von Mises stress joint pattern in the research model was $115 \mathrm{MPa}$ during screw tightening, and was reduced to $108 \mathrm{MPa}$ with removal of the wrench.
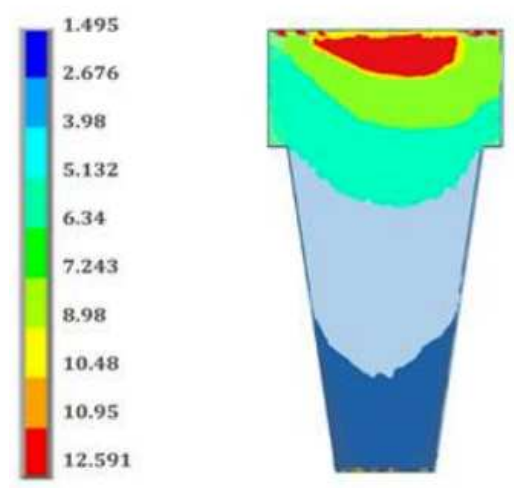

Fig. 4. Stress distribution in implant. 


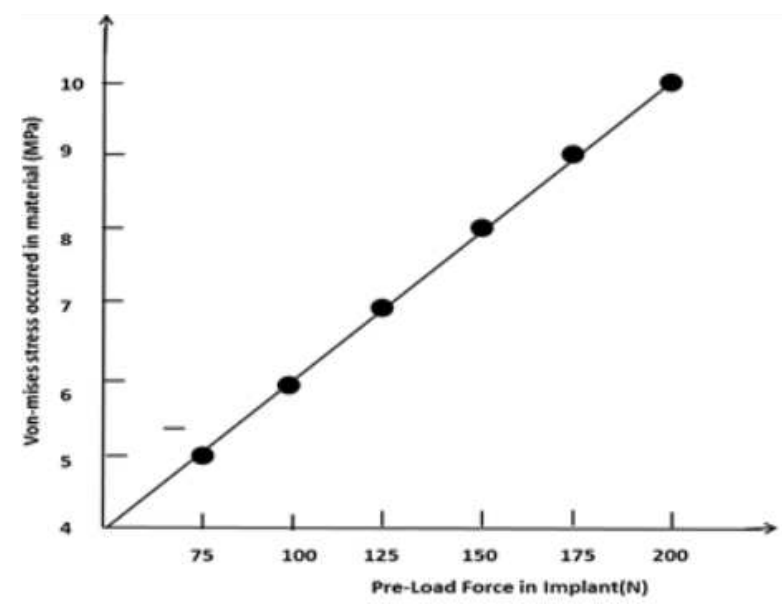

Fig. 5. Von Mises stress in implant under different forces.

The axisymmetric approach, where a cross-section can be meshed with large number of elements, provides optimal use of computing resources. The implants and abutments are modeled as titanium with linear-elastic, isotropic and homogeneous properties. Various specific stress patterns were identified within all elements of the implant complex during the assembly simulation. The numeric results showed that the equivalent von Mises stress on the mini screw under a $197 \mathrm{~N}$ tangential load reached $39 \mathrm{MPa}$ at the first thread recession, whereas von Mises stress in the peri-implant bone only reached $14 \mathrm{MPa}$ below the neck. High tightening loads of $185 \mathrm{~N} \mathrm{~mm}$ of torsion and $317 \mathrm{~N}$ of axial compression resulted in much greater bone and implant von Mises stresses than tangential loading, exceeding the yield strengths of the titanium alloy and the bone.

\section{Conclusions}

Dental implants are valuable devices for restoring lost teeth. Implants are available in many shapes, sizes and length, using a variety of materials with different surface properties. Biocompatible titanium and titanium alloys materials are used for dental implants for their excellent corrosion resistance and suitable mechanical properties. A finite element model [10] was created based on the physical properties of an implant number 5 presented for all implant-abutment-bone systems in Fig. 3. The values of the von Mises stresses generated in the crown prosthesis, abutment, implant, and supporting bone were calculated. This model can be adapted as a patient-specific clinical implant tool for planning movement of one tooth or several teeth. In this study, an increase in the implant diameter decreased the maximum von Mises equivalent stress around the implant neck more than an increase in the implant length, as a result of a more favorable distribution of the simulated forces applied. Hence, it is important to control biomechanical loads on dental implants to maintain osseointegration and to promote early bone-implant interface healing.

\section{Acknowledgments}

This research was supported by Scientific Research Projects (BAP) of Istanbul University, Turkey, project No. 24089.

\section{References}

[1] R. Wang, B. Kang, L. Lang, M. Razzoog, J. Prosthet. Dent. 101, 359 (2009).

[2] S.H. Jónsdóttir, E.W. Giesen, J.C. Maltha, Europ. J. Orthodont. 34, 542 (2012).

[3] P. Koka, A. Mohapatra, P.A. Anandapandian, K. Murugesan, M. Vasanthakumar, Indian J. Dental Res. 23, 129 (2012).

[4] V. Demenko, I. Linetskiy, K. Nesvit, A. Shevchenko, J. Dent. Res. 90, 1211 (2011).

[5] T.R. Deshmukha, A.M. Kutheb, S.M. Chawarec, V. Bagariac, D.S. Ingolea, Comput. Meth. Biomech. Biomed. Eng. 15, 363 (2012).

[6] A.C. Freitas, Implant Dentistry 19, 6 (2010).

[7] W.K. Tsui, H.D.P. Chua, L.K. Cheung, Int. J. Oral Maxillofac. Surg. 41, 1427 (2012).

[8] D. Bozkaya, S. Muftu, J. Biomech. 36, 1649 (2003).

[9] A. Ziegler, L. Keilig, A. Kawarizadeh, A. Jäger, C. Bourauel, Europ. J. Orthodont. 27, 333 (2005).

[10] ANSYS Manual, ANSYS Inc., 2013, Release 11.0 Documentation for ANSYS Workbench/Analysis Settings (10 March 2013). 